\title{
Case Report \\ Unusually High Levels of CA19-9 Associated with Mature Cystic Teratoma of the Ovary
}

\author{
Monika Madaan, ${ }^{1,2}$ Manju Puri, ${ }^{1}$ Ritu Sharma, \\ Harvinder Kaur, ${ }^{1}$ and Shubha Sagar Trivedi ${ }^{1}$ \\ ${ }^{1}$ LHMC and SSKH, New Delhi 110001, India \\ ${ }^{2}$ ESIC Hospital, Manesar, Haryana 122001, India \\ Correspondence should be addressed to Monika Madaan; monikarajivgaur@gmail.com
}

Received 22 June 2014; Revised 22 July 2014; Accepted 17 August 2014; Published 8 September 2014

Academic Editor: Giampiero Capobianco

Copyright (C) 2014 Monika Madaan et al. This is an open access article distributed under the Creative Commons Attribution License, which permits unrestricted use, distribution, and reproduction in any medium, provided the original work is properly cited.

Introduction. Mature cystic teratoma is the benign tumor of the ovary. CA19-9 levels, although a marker of pancreatic malignancy, have been found to be raised in large ovarian mature cystic teratomas. Case Report. We report a case of a young female who had unusually high levels of CA19-9 in the blood associated with large ovarian mature cystic teratoma. The levels returned to normal 12 weeks after cystectomy was performed. Conclusion. This case highlights the fact that although raised tumor marker may be associated with a benign pathology thorough evaluation to rule out malignancy still must be done.

\section{Introduction}

Mature cystic teratoma or dermoid tumor is the most common tumor of young females and constitutes up to a third of all benign ovarian tumors. It is composed of welldifferentiated tissues derived from all three germ layers. It is commonly diagnosed based upon clinical findings, ultrasonography, or CT scan. CA19-9 levels are raised in patients with malignancy of pancreas, biliary tract, colon, esophagus, and liver carcinoma. There are reports in the literature documenting raised blood levels of CA19-9 in women with mature cystic teratomas but there is no report associating extremely high levels with a benign ovarian pathology. We describe a case of mature cystic teratoma associated with very high levels of CA19-9 in the blood.

\section{Case Presentation}

A 27-year-old woman, married for 9 months, P0A1, was referred to us in the gynaecology outpatient clinic with the diagnosis of ovarian cyst on USG and CA19-9 level of $1826 \mathrm{U} / \mathrm{mL}$. She complained of heaviness of the lower abdomen and gradually developing distension for the past 4 months. Her menstrual cycle was normal and past history was not significant. On examination, her vitals were stable and abdominal examination revealed a large intra-abdominal mass reaching up to the umbilicus. Vaginal examination revealed normal sized retroverted uterus deviated to the right. The mass felt per abdomen could be tipped through anterior and left fornix. The movements of the mass were not transmitted to the cervix. In investigations, her hematological and biochemical profile was normal. Transabdominal USG showed a complex cystic lesion measuring $15 \times 13 \times 9.5 \mathrm{~cm}$ in the left ovary with internal echoes and septae, giving the possibility of mature cystic teratoma. CT scan also reported the cyst as left ovarian dermoid. The other abdominal and pelvic organs were unremarkable. In view of abnormally high values of CA19-9 in association with ovarian cyst other tumor markers like CA125, AFP, LDH, and beta hCG were measured and were found to be normal.

Patient was taken up for exploratory laparotomy. A large left sided ovarian mature cystic teratoma measuring around $15 \times 12 \mathrm{~cm}$ was present. Uterus, bilateral tubes, and right ovary were normal. Left sided ovarian cystectomy was done. The cyst was removed intact; there was no extracapsular extension, no spread to the other ovary, and no ascites. A thorough exploration of the whole abdomen was carried out and no abnormality was detected. Frozen section of the cyst 
was reported as mature cystic teratoma and the tumor was staged as stage 1. CA19-9 levels decreased to $975 \mathrm{U} / \mathrm{mL} 5$ days after surgery and finally returned to normal 2 months following surgery.

\section{Discussion}

CA19-9 is a sialylated Lewis blood group antigen which is primarily used for diagnosis, follow-up, and prognosis of pancreatic carcinoma. Its normal value is $<35 \mathrm{U} / \mathrm{mL}$. It is considered as the most sensitive and specific marker of pancreatic carcinoma. Its level is elevated in malignancies of biliary tract, colon, esophagus, and liver. Certain benign conditions like pancreatitis, biliary disease, and cirrhosis are also associated with elevated levels $>1000 \mathrm{U} / \mathrm{mL}$ [1]. However $5-7 \%$ of population is Lewis antigen negative and in them CA19-9 levels are undetectable in the blood irrespective of the pathology [1].

Increased levels of CA19-9 have been observed in some cases of mature cystic teratoma. Atabekoglu et al. described a case report with CA19-9 value of $1430 \mathrm{U} / \mathrm{mL}$ with dermoid cyst [2]. Their study also showed that it is secreted from the apical cytoplasm of the epithelial lining of the cyst wall as is shown by immunohistochemical staining resulting in detectable levels found in systemic circulation. Abnormally high levels have been found in very large mature cystic teratomas probably associated with inflammation of the cyst wall or a weakened cyst wall. This also explains very high levels found in our case as the size of the cyst was quite large and also the reason for the long time taken for the levels to return to normal. Coskun et al. described mean levels of $109.1 \mathrm{U} / \mathrm{mL}$ in their case series of 43 patients with an average diameter of $7.7 \mathrm{~cm} \mathrm{[3].} \mathrm{Kikkawa} \mathrm{et} \mathrm{al.} \mathrm{found} \mathrm{the} \mathrm{mean} \mathrm{level} \mathrm{of}$ $217.6 \mathrm{U} / \mathrm{mL}$ in their series of 71 cases [4].

Brain et al. described markedly high levels of $2880 \mathrm{U} / \mathrm{mL}$ in a case with ruptured ovarian cyst (mucinous cystadenoma) associated with ascites [5]. Studies have shown that CA19-9 could be increased in up to $50 \%$ of cases of immature cystic teratoma [6]. It can be used as a marker for postoperative follow-up in benign disease and as a marker for recurrence of mature cystic teratoma.

\section{Conclusion}

To conclude, extremely high levels of CA19-9 may be associated with benign ovarian pathology, but thorough preoperative workup for any evidence of malignancy is a must.

\section{Consent}

Written informed consent was obtained from the patient for publication of this case report.

\section{Conflict of Interests}

The authors declare that there is no conflict of interests regarding the publication of this paper.

\section{References}

[1] W. Steinberg, "The clinical utility of the CA 19-9 tumorassociated antigen," The American Journal of Gastroenterology, vol. 85, no. 4, pp. 350-355, 1990.

[2] C. Atabekoglu, E. A. Bozac, and S. Tezcan, "Elevated carbohydrate antigen 19-9 in a dermoid cyst," International Journal of Gynecology and Obstetrics, vol. 91, no. 3, pp. 262-263, 2005.

[3] A. Coskun, G. Kiran, and O. Ozdemir, "CA 19-9 can be a useful tumor marker in ovarian dermoid cysts," Clinical and Experimental Obstetrics and Gynecology, vol. 35, no. 2, pp. 137139, 2008.

[4] F. Kikkawa, A. Nawa, K. Tamakoshi et al., "Diagnosis of squamous cell carcinoma arising from mature cystic teratoma of the ovary," Cancer, vol. 82, no. 11, pp. 2249-2255, 1998.

[5] O. Brain, L. H. W. Brown, S. Suvarna, and R. Chapman, "Markedly elevated CA19-9 associated with benign ovarian cyst and ascites," BMJ Case Reports, vol. 2009, 2009.

[6] S. Novaković, "Tumor markers in clinical oncology," International Journal of Radiation Oncology, vol. 38, no. 2, pp. 73-83, 2004. 


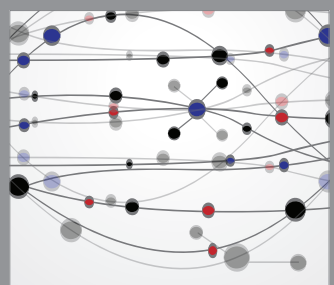

The Scientific World Journal
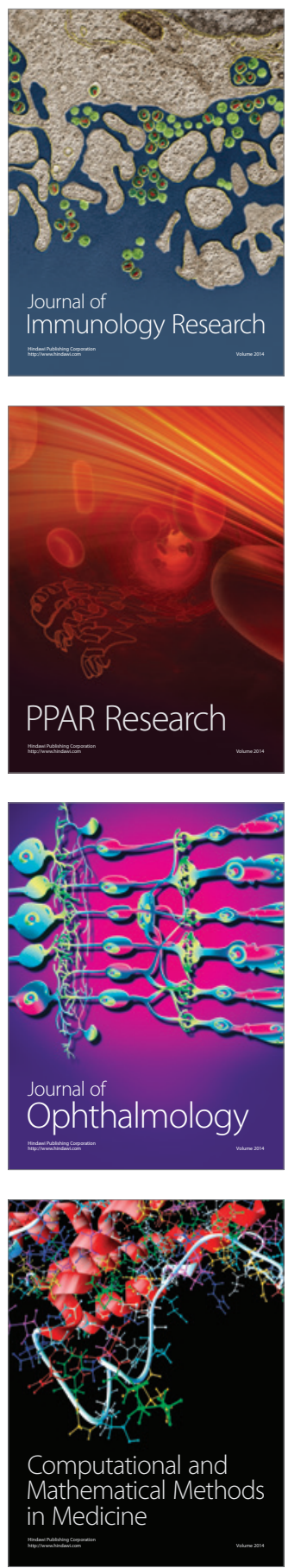

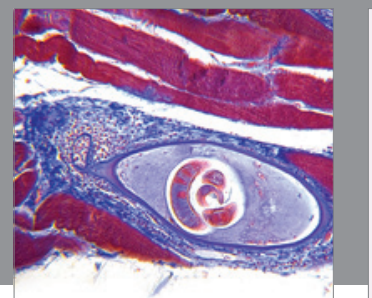

Gastroenterology

Research and Practice
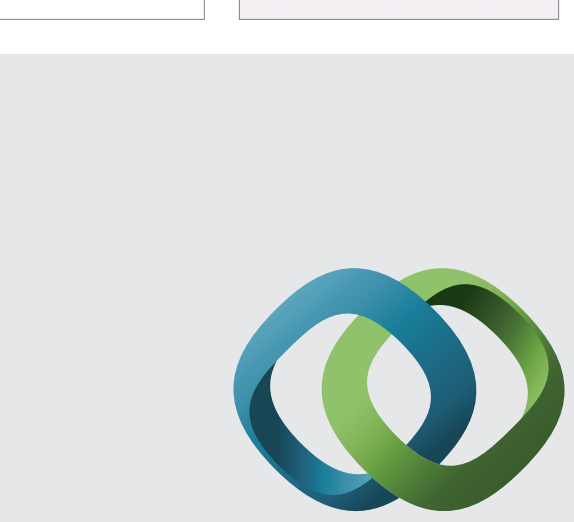

\section{Hindawi}

Submit your manuscripts at

http://www.hindawi.com
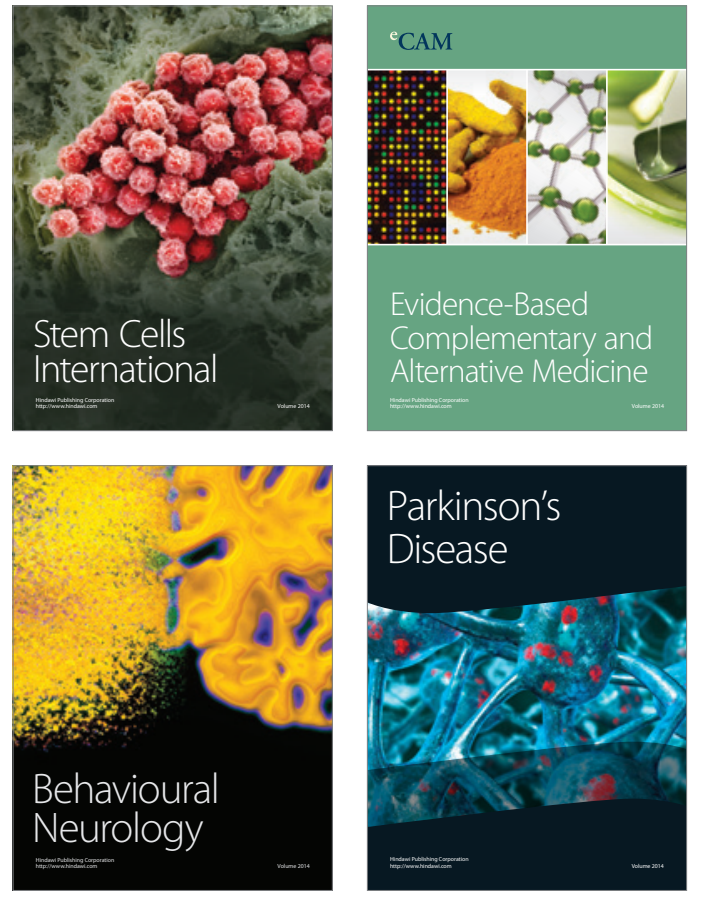
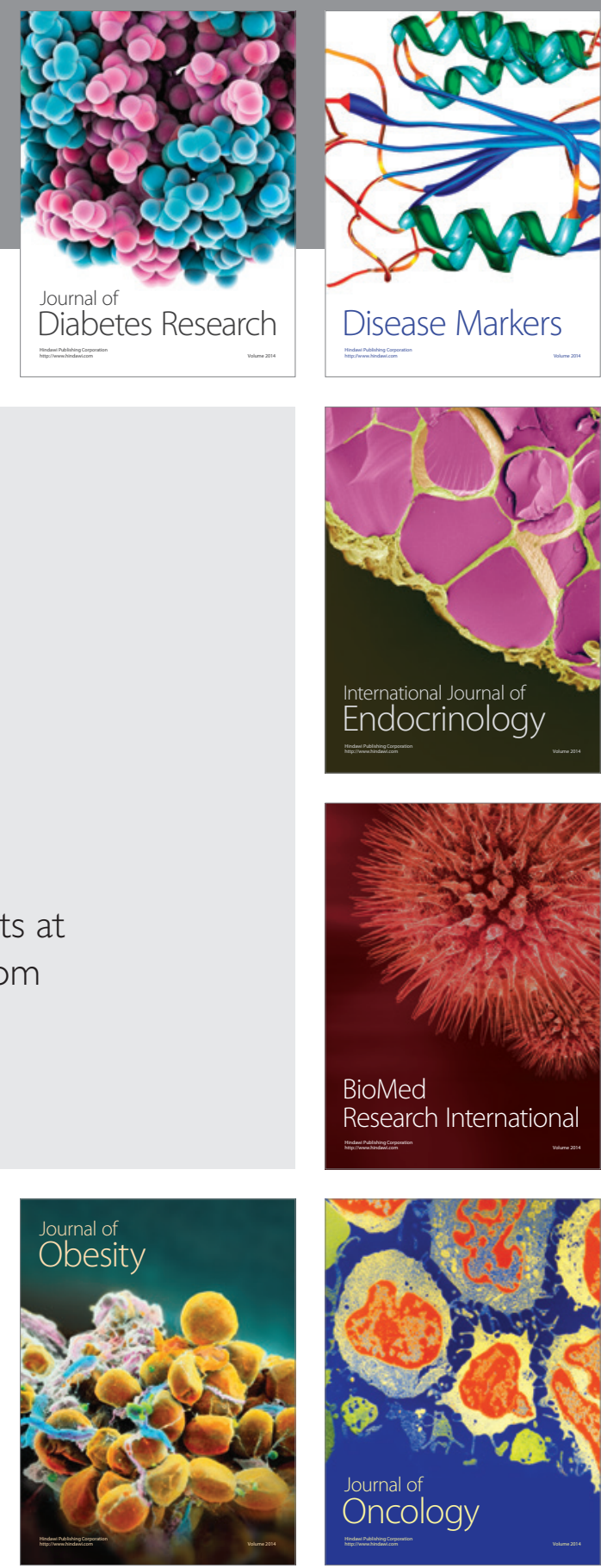

Disease Markers
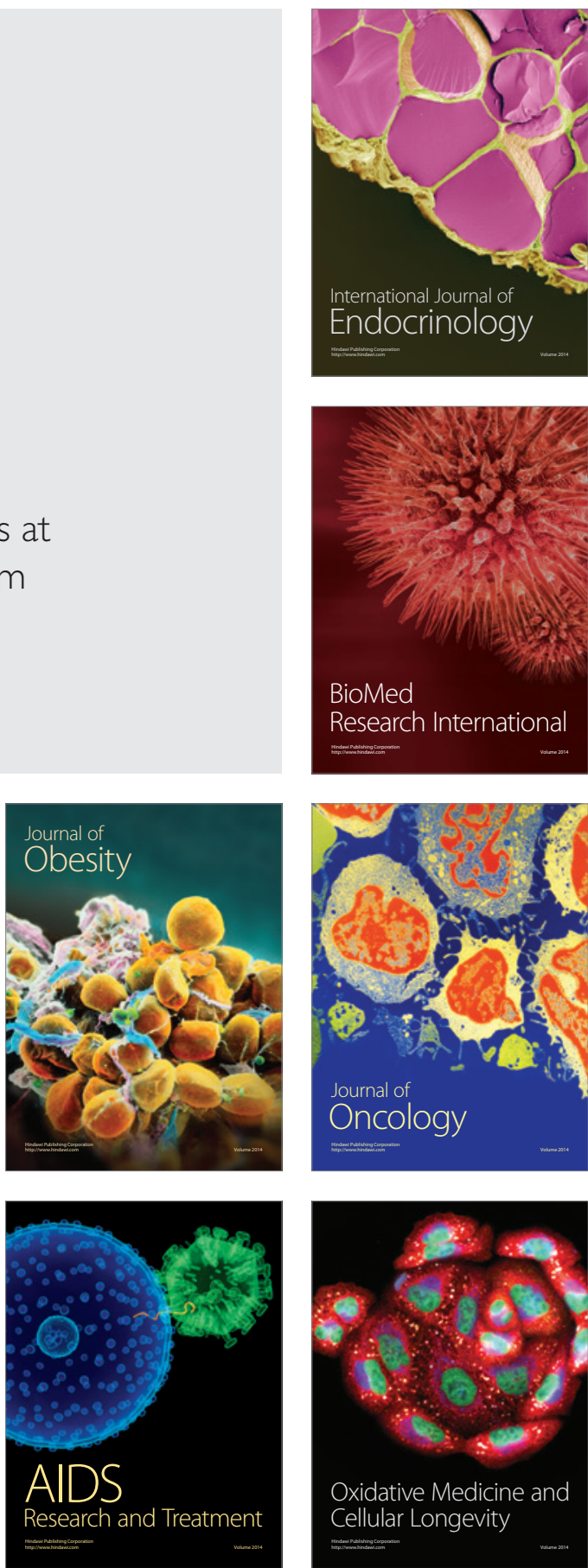